\title{
Topological study of refractive indices, atomic charges, polar and axial vectors of second rank and optical rotation in $\alpha$-HgS (cinnabar) at $296 \mathrm{~K}$.
}

\section{Karppinen}

Institute of Chemistry, University of Uppsala, Box 531, Uppsala S-75 121 Sweden.

Correspondence e-mail: markkuk96@gmail.com

\begin{abstract}
The magnitude and sense of optical rotation are determined from the point charge model in a nonpolar, chiral, covalently bonded and semiconducting $\alpha$-HgS (cinnabar) crystal. Atomic charges of $\mathrm{Hg}$ and $\mathrm{S}$ are variables in the iteration of topological equivalence between the inverted ratios of the second electric moments and the corresponding optical refractive indices of the $\alpha-\mathrm{HgS}$ crystal. Vector cross products of the wave vector in the propagation direction of light and the second electric moments in the other two semi-axis directions of the quadric specify the handedness of axial vectors of second rank and the refractive indices comprise the magnitudes of them. The calculated axial vectors contain information of optical rotation and the handedness of the dominant component of them reveals the sense of rotational character of the crystal. They are converted to principal gyration tensor components and the magnitude and sense of rotation are computed in the direction of optic axis of the quadric. The sense of optical rotation is opposite to the sense of the helical arrangement of the atoms. The magnitude and sense of optical rotation solely arises from the asymmetric distribution of point charges and
\end{abstract}


electric vectors in the unit cell of $\alpha-\mathrm{HgS}$ crystal. The morphological right- and left-handed character of the two enantiomorphs and the absolute structure of $\alpha-\mathrm{HgS}$ crystal are discussed.

Keywords: $\alpha$-HgS (cinnabar),refractive indices,atomic charges, polar and axial vectors of second rank,optical rotation

\section{Introduction}

The atoms can easily be seen arranged in helices in many of the enantiomorphic crystals in tetragonal, trigonal and hexagonal symmetries and it has been suggested that the helical arrangement of atoms must be a requirement for the existence of optical rotation. One of the first observations of the opposite sense of optical rotation was made in the right- and lefthanded crystals of quartz. They are enantiomorphic pairs and mirror images of each other with the opposite screw rotational helices of atoms in their respective space groups. They are built up of $\mathrm{SiO}_{2}$ units connected together in spiral-helices. Fresnel [1] supposed that, when a planepolarized light enters the optically active crystal in an optic axis direction, it splits up into two circularly polarized waves with opposite rotations but with the same frequency. The classical electromagnetic theory of oscillating electric charges, which are coupled together, was developed under the leadership of Max Born. [2] It resulted in good agreement with the experimental observations when applied in $\alpha$-quartz. [3] It has later been summarized by Condon that the existence of coupled oscillators is not essential to obtain the acceptable results of optical rotation. [4] The interpretation of optical rotation in crystal is still often connected to the helical arrangement of atoms in crystals.

Mercury, $\mathrm{Hg}$, forms compounds with the oxidation numbers (I) and (II), of which $\mathrm{Hg}$ (II) is more common. $\mathrm{Hg}$ (II) nitrate, sulfate and perchlorate salts are totally ionic and they dissociate in a water solution. A considerable covalent character in the mercury-ligand bonds is found in the two-coordinated compounds of $\mathrm{Hg}(\mathrm{II})$ with $\mathrm{C}, \mathrm{N}, \mathrm{P}$ and $\mathrm{S}$ as ligand atoms. The $\mathrm{Hg}$ has a full electron occupancy on the $5 \mathrm{~d}$-orbital $\left(5 \mathrm{~d}^{10}\right)$, but its $6 \mathrm{~s}^{2}$ electrons can overlap with $3 \mathrm{~s}^{2} \mathrm{p}^{4}$ electrons of S and form linear hybrid orbitals for bonding according to the theories after Cotton and Wilkinson. [5] Three crystalline phases of $\mathrm{HgS}$ are known. $\alpha$-HgS (cinnabar) is a transparent red mineral, which is insoluble in water. It is most stable mineral of $\mathrm{HgS}$ at room 
temperature and it crystallizes in the trigonal symmetry class 32 . It has earlier been used as a pigment, which has been found to darken in a long run due to a slow phase transition to $\beta$ - $\mathrm{HgS}$ (metacinnabar). Black metacinnabar is a higher temperature phase of $\mathrm{HgS}$ in a cubic symmetry class $43 \mathrm{~m}$. Both $\alpha-\mathrm{HgS}$ and $\beta$ - $\mathrm{HgS}$ have relative low band-gap energies of 2.0 and $1.6 \mathrm{eV}$, respectively, which have made them suitable for photoelectric and semiconductor materials today. The former absorbs light at $620 \mathrm{~nm}$. [6] Hypercinnabar has a hexagonal symmetry and it requires even a higher temperature than metacinnabar to crystallize. The space group of it is unknown.

The structure of $\alpha$-HgS has been determined in the space group $P 3_{2} 21$ by anomalous X-ray scattering. [7],[8] The 32 screw rotates anticlockwise away from the viewer and clockwise towards the viewer in the optic axis direction. Light was tested to rotate opposite to the helix of the atoms, when the Faraday rotation and the optical rotatory power of synthetic $\alpha-\mathrm{HgS}$ were measured close to the absorption edge with $\lambda=6328 \AA$. [9] The rotation of $-320^{\circ} \mathrm{mm}^{-1}$ was observed in the unique axis direction of the cinnabar crystal in a study, which was based on the contour diagram of the variation of the calculated rotatory power in a function of assumed atomic polarizabilities of $\mathrm{Hg}$ and $\mathrm{S}$. [9], [10] It was shown later that two symmetric and one non-symmetric structural helices of $\mathrm{S}$ atoms follow the sense of the screw rotation in the space group $P 3_{2} 21$ and contribute with the same sense and magnitude to the total optical rotation in the optic axis direction of $\alpha$-HgS crystal in agreement with the experimental observation, when zero polarizabilities were given to $\mathrm{Hg}$ atoms. [11]

This study presents an alternative way to describe optical rotation in crystals basing on the fact that axial vectors, which have a screw rotational character, can explain with their handedness the rotational sense of the circularly polarized light waves, when they travel in the optically active crystal. The gyration tensor is one example of the axial tensor of second rank and hence the gyration tensor components are axial vectors of second rank. They contain information about optical rotation of light in crystals. [12] In order to get accurate information about axial vectors, the crystal structure, atomic net charges and optical refractive indices, all measured under the same circumstances, must be known.

The total electrostatic potential is a sum of the individual potentials at any point out of the system of point charges and it can be developed into a power series in the Cartesian coordinate system. [13],[14] The power series contains the components of the zeroth, first, second, third, 
fourth etc. electric moments in the principal axis directions of the crystal. The first and third electric moments have odd parity and the zeroth, second and fourth moments have even parity. Odd parity moments change the sign, while the even parity moments have the same sign on inversion. Interaction between light and electric moments of second rank in crystals forms an optical indicatrix, which is a quadric (= a surface of second rank), where the refractive indices are mutually orthogonal semi-axes of it. They are positive quantities and have a quadradic character and even parity. A quadric as a representation surface can even be designed for the multi-nuclear system of point charges with the second electric moments as mutually perpendicular semi-axes. The second electric moments have also an even parity due to the squared atomic coordinates. The indicatrix is a macro-quadric, while the surface of second electric moments originating from the unit cell is a nano-quadric. They both can be presented as ellipsoids of revolution in the tetragonal, trigonal and hexagonal crystal symmetries and they can be surveyed after interesting topological equivalences between their parameters through mathematical iterations.

Because $\alpha-\mathrm{HgS}$ is a covalently bonded semiconductor, it can be characterized as a molecular compound, where the rule of integer charges is not obeyed. The $\mathrm{Hg}(\mathrm{II})$ and S share the bonding electrons and have equal but opposite charges. The charges are variables in the iteration, which is stopped, when the inverted ratio of the calculated second electric moments reaches the ratio of the corresponding refractive indices. The cross products between the polar wave vector of light and the polar vectors of second electric moments in a crystal generate axial vectors of second rank. In this study the structural and optical chirality are discussed in terms of the sense of helical chains of $\mathrm{Hg}$ and $\mathrm{S}$ atoms and the rotational sense of the dominant components of the axial vectors. The magnitude of optical rotation is the difference between the two axial vectors of the quadric and the theory proposed by Fresnel is applied to confirm the sense of optical rotation in the two enantiomorphs of $\alpha-\mathrm{HgS}$ crystal in the space groups $P 3_{1} 21$ and $P 3_{2} 21$.

\section{Structural basis for calculations}

The lattice parameters and the refined atomic positions of $\alpha-\mathrm{HgS}$ in the space group $P 3_{2} 21$ are taken from Refs. [7],[8] and they are standing in TABLE I together with the coordinates of the atoms in one chain, which are derived from the symmetry cards (S.G. 154); X,Y,Z, -Y,X$\mathrm{Y}, \mathrm{Z}+2 / 3, \mathrm{Y}-\mathrm{X},-\mathrm{X}, \mathrm{Z}+1 / 3, \mathrm{Y}, \mathrm{X},-\mathrm{Z}, \mathrm{X}-\mathrm{Y},-\mathrm{Y}, 1 / 3-\mathrm{Z},-\mathrm{X}, \mathrm{Y}-\mathrm{X}, 2 / 3-\mathrm{Z}$. The crystal structure contains 
continuous -Hg-S-Hg-S-Hg-S- spiral chains, which run in the unique $c$-axis direction in each corner of the hexagonal unit cell. The Hg-S-Hg and the S-Hg-S angles are $104.7^{\circ}$ and $172.8^{\circ}$, respectively. The shortest Hg-S distances are $2.368 \AA$ in a chain and $3.094 \AA$ between the chains. In the crystallographic calculations atomic positional coordinates must be greater than 0 and less than 1 . The second electric moments have exponential values of atomic coordinates and they are only comparable with each other, when the atoms are transformed to the positions with an equivalent reference origin. Hence all three axes are doubled resulting at new atomic coordinates according to $\mathrm{X}=(\mathrm{X}+1) / 2, \mathrm{Y}=(\mathrm{Y}+1) / 2$ and $\mathrm{Z}=\mathrm{Z} / 2$. The application of initial electric charges +1 for $\mathrm{Hg}$ and -1 for $\mathrm{S}$ transforms the atoms to the final coordinates with their centers of charge at the coordinate $(=1 / 2)$. They are written in TABLE II. Two plots of the crystal structure of $\alpha-\mathrm{HgS}$ in the space group $P 3_{2} 21$ are displayed in Figs. 1(a) and 1(b). [15]

TABLE I. Lattice parameters and the refined atomic coordinates together with the position coordinates of the atoms in one chain.

\begin{tabular}{|c|c|c|c|c|}
\hline \multicolumn{3}{|c|}{$a=b=4.145 \AA$} & \multicolumn{3}{c|}{$c=9.496 \AA$} \\
\hline Atom & & $\mathrm{X}$ & $\mathrm{Y}$ & $\mathrm{Z}$ \\
\hline $\mathrm{Hg}$ & Refined & 0.7198 & 0 & $2 / 3$ \\
\hline $\mathrm{S}$ & “ & 0,4889 & 0 & $1 / 6$ \\
\hline $\mathrm{S} 1$ & & \multicolumn{3}{|c|}{ Atomic coordinates in one chain } \\
\hline $\mathrm{Hg} 1$ & & 0.4889 & 0 & $1 / 6$ \\
\hline $\mathrm{S} 2$ & & 0 & -0.2802 & $2 / 6$ \\
\hline $\mathrm{Hg} 2$ & & -0.2802 & -0.4889 & $3 / 6$ \\
\hline $\mathrm{S} 3$ & & 0 & 0 & $4 / 6$ \\
\hline $\mathrm{Hg} 3$ & & 0.2802 & 0.4889 & $5 / 6$ \\
\hline $\mathrm{S} 4$ & & 0.4889 & 0.2802 & $6 / 6$ \\
\hline
\end{tabular}

TABLE II. Transformed atomic coordinates.

These coordinates are used in the iteration of the net atomic charges and in the calculations of the electric moments.

\begin{tabular}{|c|c|c|c|}
\hline Atom & $\mathrm{X}$ & $\mathrm{Y}$ & $\mathrm{Z}$ \\
\hline $\mathrm{S} 1$ & $1 / 2$ & $1 / 2$ & $3 / 12$ \\
\hline $\mathrm{Hg} 1$ & 0.25555 & 0.35990 & $4 / 12$ \\
\hline
\end{tabular}




\begin{tabular}{|c|c|c|c|}
\hline $\mathrm{S} 2$ & 0.01110 & 0.25555 & $5 / 12$ \\
\hline $\mathrm{Hg} 2$ & 0.11545 & $1 / 2$ & $6 / 12$ \\
\hline $\mathrm{S} 3$ & 0.25555 & 0.74445 & $7 / 12$ \\
\hline $\mathrm{Hg} 3$ & 0.39565 & 0.64010 & $8 / 12$ \\
\hline $\mathrm{S} 4$ & $1 / 2$ & $1 / 2$ & $9 / 12$ \\
\hline & $\Sigma e_{i} x_{i}=-0.5$ & $\Sigma e_{i} y_{i}=-0.5$ & $\Sigma e_{i} z_{i}=-0.5$ \\
& $\Sigma e_{i}=-1$ & $\Sigma e_{i}=-1$ & $\Sigma e_{i}=-1$ \\
& $x_{c}=0.5$ & $y_{c}=0.5$ & $z_{c}=0.5$ \\
\hline
\end{tabular}

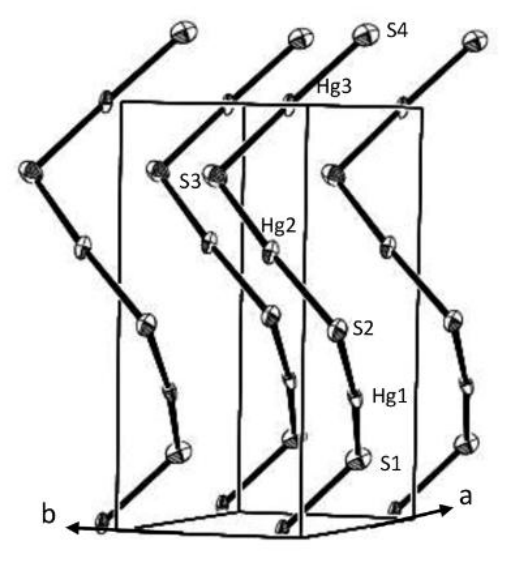

1(a)

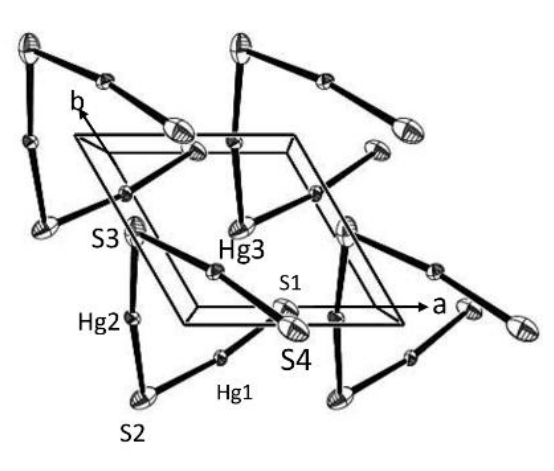

1(b)

Figure 1. The crystal structure of $\alpha-\mathrm{HgS}$ in space group $P 3_{2} 21$ is designed in two displacement ellipsoid plots. [15] Fig. 1(a) shows the four chains of atoms running in the $c$-axis direction and Fig. 1(b) a view to (001) of the unit cell. In both figures one chain with the numbered atoms follows the coordinates in TABLE II.

\section{Iteration of atomic charges and electric moments}

$\alpha-\mathrm{HgS}$ has an ideal crystal structure for the determination of optical rotation. It is a simple compound containing covalently bonded atoms in the continuous helical chains in the optic axis direction of crystal with weaker covalent bonds between the chains. The clockwise rotation sense of the atomic helices towards the observer is directly readable from the crystal structure in Fig. 1(b). When the four neighbor cells share the rotational information around each corner in a hexagonal unit cell, the atomic parameters of only one chain in the unit cell are needed in the following calculations. The repeat distance $c$ in Fig.1(a) and the complete 
atomic helix in Fig.1(b) require that the bonds between seven atoms, which give the chain a negative charge, must be included in the computations.

The Eq. (1) is an expression for a principal net component, $\Theta_{\mathrm{xx}}^{\prime}$, of the second electric moment in the $a$-axis direction. It is developed for multinuclear negative ions in ionic solids and presented in the study of $\mathrm{LiNaSO}_{4}$ [17]. It worked well also in a semiconductor $\alpha-\mathrm{TeO}_{2}$. [18]

$$
\Theta_{\mathrm{xx}}^{\prime}=\Sigma e_{i} x_{i}^{2}-2 \mu_{x} x_{c}+q X^{2}
$$

where $e_{i}$ is a charge of an atom with a coordinate $x_{i} . x_{c}$ is the location of the first electric moment $\mu_{x}\left(=\Sigma e_{i} x_{i}\right) . \quad q$ is a total charge of the ion and $X^{2}$ is a square of its position coordinate, which must be separately defined. There is no first electric moment in the crystallographic $c$ axis direction of $\alpha-\mathrm{HgS}$ crystal, due to symmetry, though the bonds between $\mathrm{Hg}$ and $\mathrm{S}$ have a polar covalent character. But the first electric moments with equal magnitudes in the $a$ - and $b$ axis directions are existing. The expression for it can be utilized in the determination of the value of $X^{2}$ in Eq. (1), where the charge is normalized to +1 . The following equality according to Eq. (2) holds for a polar vector in the $a$-axis direction of $\alpha$-HgS crystal:

$$
\begin{aligned}
& q x_{c}-q X^{2}=q \cdot(-0.5) \\
& \Rightarrow \quad-q X^{2}=-1 \cdot 0.5-q x_{c} \\
& \Rightarrow \quad q X^{2}=1 \cdot 0.5+q x_{c} \\
& \Rightarrow \quad X^{2}=\left(1 \cdot 0.5+q x_{c}\right) / q,
\end{aligned}
$$

where $q$ is a positive charge of Hg-atom. $x_{c}$ is the coordinate of the center of the charge $(=1 / 2)$, (in TABLE II). $q X^{2}>q x_{c} . x_{c}$ and the first electric moment $(=-0.5)$ with the charge normalized to +1 , are constants and independent of the value of $q$. The only requirement is that $q>1$ then $X^{2}<1$ and the explored value of $X^{2}$ can be used to get iteration to work in the calculation of net atomic charges and second electric moments.

The following three equations are the components of the second electric moment in a nanoquadric of $\alpha-\mathrm{HgS}$ crystal. They are multiplied by the squared doubled lattice constants and divided by 8 to get out the unit cell information. Due to a hexagonal symmetry the equations (3) and (4) are multiplied by $\cos ^{2}(30)$ to reorient them as mutually orthogonal semi-axes and used together with the Eq. (2) in the iteration with the atomic charges of $\mathrm{Hg}$ and $\mathrm{S}$ as variables. Due to symmetry $Y^{2}=X^{2}$, but in the nonpolar $c$-axis direction $Z^{2}=0.5^{2}$. The atomic positional 
parameters are taken from TABLE II. $q$ is the total negative charge of the ion and $\mu_{x}=\Sigma e_{i} x_{i}, \mu_{y}=$ $\Sigma e_{i} y_{i}$ and $\mu=\Sigma e_{i} z_{i}$, are calculated with the charges given in TABLE III.

$$
\begin{aligned}
& \Theta_{\mathrm{xx}}^{\prime}=\left[\left(\sum e_{i} x_{i}^{2}-2 \mu_{x} x_{c}+q X^{2}\right) \cdot \cos ^{2}(30) \cdot(2 \cdot a)^{2}\right] / 8 \mathrm{e} \AA^{2} \\
& \Theta_{\mathrm{yy}}^{\prime}=\left[\left(\sum e_{i} y_{i}^{2}-2 \mu_{y} y_{c}+q Y^{2}\right) \cdot \cos ^{2}(30) \cdot(2 \cdot b)^{2}\right] / 8 \mathrm{e} \AA^{2} \\
& \Theta_{\mathrm{zz}}^{\prime}=\left[\left(\sum e_{i} z_{i}^{2}-2 \mu_{z} z_{c}+q Z^{2}\right) \cdot(2 \cdot c)^{2}\right] / 8 \mathrm{e} \AA^{2}
\end{aligned}
$$

The reliable dispersion formulas of refractive indices, $n_{e}$ and $n_{o}$, for $\alpha-\mathrm{HgS}$ within the wavelength range of 0.62 and $11 \mu \mathrm{m}$ are taken from the Ref. [16] The values $n_{e}=3.232203$ and $n_{o}=2.885090$ are reckoned with $0.6328 \mu \mathrm{m}$. The ratio $n_{e} / n_{o}=1.120313$ is used as a reference in the iteration as denoted in TABLE III.

TABLE III. A summary of the input and obtained parameters in the iteration.

\begin{tabular}{|c|c|c|c|c|c|c|}
\hline $\mathrm{Hg}$ & $\mathrm{S}$ & $\mathrm{X}^{2}=\mathrm{Y}^{2}$ & $\Theta_{\mathrm{xx}}^{\prime}=\Theta_{\mathrm{yy}}^{\prime} \mathrm{e}^{2}$ & $\Theta^{\prime}{ }_{\mathrm{zz}} \mathrm{e}^{2}$ & $\Theta_{\mathrm{xx}} / \Theta_{\mathrm{zz}}$ & $n_{e} / n_{0}$ \\
\hline+1 & -1 & 1 & -5.349240 & -3.757251 & 1.423711 & \\
\hline+2 & -2 & 0.75 & -7.477039 & -7.514501 & 0.995015 & \\
\hline+1.5 & -1.5 & 0.833333 & -6.413140 & -5.635876 & 1.137994 & \\
\hline+1.54 & -1.54 & 0.824675 & -6.498252 & -5.786166 & 1.123067 & \\
\hline+1.55 & -1.55 & 0.822581 & -6.519530 & -5.823739 & 1.119475 & \\
\hline+1.5476 & -1.5476 & 0.823081 & -6.514423 & -5.814721 & 1.120333 & 1.120313 \\
\hline
\end{tabular}

It was discovered in the study of trigonal $\mathrm{LiNaSO}_{4}$ that the ratios of the refracted indices and the corresponding second electric moments, derived from the refined net atomic charges under ionic constraints in an asymmetric unit of the crystal, were invert numbers. [17] The same situation appeared also between the iterated moments and refractive indices in $\alpha-\mathrm{TeO}_{2}$ and in $\alpha-\mathrm{LiIO}_{3}$ crystals. [18], [19] The reciprocal correlation can only be explained that the measured refractive indices, $n_{e}$ and $n_{o}$, contain information of the axial vectors of second rank, arising from the cross products between the wave vector of light acting in one axis direction and the second electric moments derived from the other two principal axis directions of the nanoquadric, when a light beam traverses the crystal towards the observer. Two quadrics (not scaled) are designed in Figure 2., where the inverted ratio of the iterated polar second electric moments in Fig.2(a) are standing in the topological equivalency (Encyclopedia Britannica) with the corresponding ratio of the refractive indices in Fig. 2(b). 


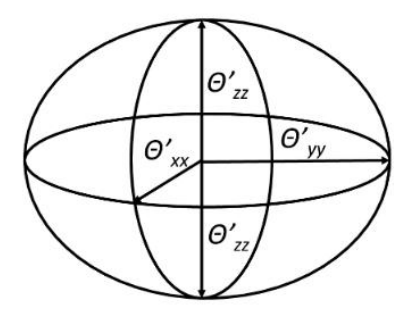

2(a)

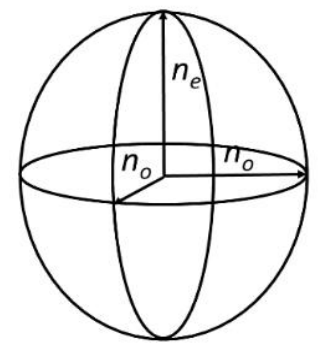

2(b)

Figure 2. Two quadrics (not scaled) of: 2(a) the polar second electric moments and 2(b) the optical indicatrix of $\alpha-\mathrm{HgS}$ crystal with the topologically equivalent ratios of the orthogonal semi-axes. The two objects are determined with different techniques from the $\alpha-\mathrm{HgS}$ crystal and they don't share any numerical parameters.

\section{Refractive indices and handedness of axial vectors of second rank}

$\alpha$-HgS is optically positive $\left(n_{e}>n_{o}\right)$ chiral compound. Only two possible structural descriptions of the $\alpha-\mathrm{HgS}$ crystal are existing, because the symmetry class 32 is nonpolar. They are two enantiomorphic crystals, which are morphological, structural and optical mirror images of each other in space groups $P 3_{1} 21$ and $P 3_{2} 21$. One of them is a right-handed and the second one a left-handed crystal. One of them has the absolute crystal structure, which can be tested against the accurately collected and entire X-ray dataset. The absolute structure of $\alpha-\mathrm{HgS}$ crystal is not known, but the absolute configuration of it in space group $P 3_{2} 21$ was tested to be levorotatory. [8] It helps to verify the absolute structure of the crystal, when the handedness of the dominant axial vector of the tested absolute configuration is defined.

A monochromatic unpolarized light traveled in the crystallographic $a$-axis direction of a righthanded crystal, when the refractive indices of $\alpha-\mathrm{LiIO}_{3}$ crystal were measured. [18] A similar treatment can be used to determine the handedness of the axial vectors of second rank in the measurement of optical refractive indices of $\alpha-\mathrm{HgS}$ crystal. The atomic coordinates of the ionic model used in this study are presented in TABLE II. The chain has a negative first electric moment, which is pointing in a negative $c$-axis direction and it is reasonable to let the second electric moment point in the same direction. In the following presentations $\mathbf{k}_{\mathbf{a}}$ is a wave vector of light and the polar second electric moments, $\Theta^{\prime}{ }_{x x}, \Theta^{\prime}{ }_{y y}$ and $\Theta^{\prime}{ }_{z z}$, are denoted by $\mathbf{A}$ and $\mathbf{B}$ in the positive and $\mathbf{C}$ in the negative direction of semi-axes of the nano-quadric. The axial vectors 
are marked by $\mathbf{A}^{\prime}, \mathbf{B}^{\prime}$ and $\mathbf{C}^{\prime}$. In the following illustrations the rotational sense of the axial vectors is defined, how a circularly polarized light will rotate in them, when it propagates away from the origo in a right- and left-handed crystals of $\alpha$-HgS. Figs. 3(a) and 3(b) present the measurements of the ordinary $\left(n_{o}\right)$ and extraordinary $\left(n_{e}\right)$ refractive indices in a right-handed crystal. Figs. 3(c) and 3(d) illustrate the measurements in a left-handed crystal. All figures are designed in a right-handed system of coordinate axes.

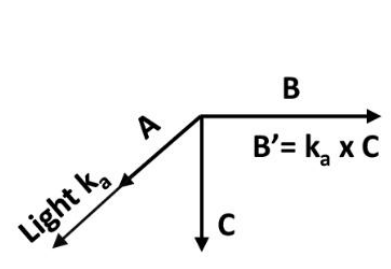

3(a)

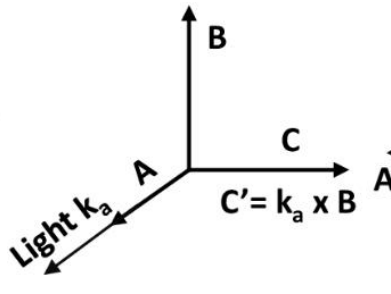

3(b)

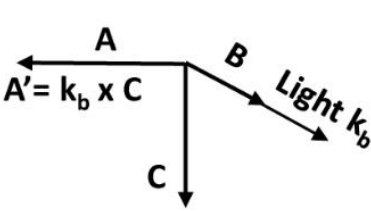

$3(\mathrm{c})$

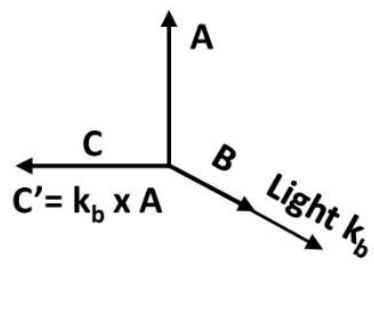

3(d)

Figure 3. Wave vector of light, second electric moments and axial vectors. Figs. 3(a) and 3(b) in a righthanded and Figs.3(c) and 3(d) in a left-handed $\alpha$-HgS crystal.

Light propagates in the $a$-axis direction of a right-handed $\alpha-\mathrm{HgS}$ crystal in Fig. 3(a), when the refractive index is read in the $\mathbf{B}$ axis direction of the nano-quadric. The vectors $\mathbf{k}_{\mathbf{a}}, \mathbf{C}$ and $\mathbf{B}^{\prime}$ follow right-hand rule in the axial vector $\mathbf{B}^{\prime}=\mathbf{k}_{\mathbf{a}} \mathbf{x} \mathbf{C}$. The circularly polarized light will rotate clockwise $\left(n_{R}\right)$ in $\mathbf{B}^{\prime}$. The crystal is reoriented to read the index in the $\mathbf{C}$ axis direction in Fig. 3(b), where the vectors $\mathbf{k}_{\mathbf{a}}, \mathbf{B}$ and $\mathbf{C}^{\prime}$ follow left-hand rule and give the dominant axial vector $\mathbf{C}^{\prime}=\mathbf{k}_{\mathbf{a}} \mathbf{x} \mathbf{B}$ a counterclockwise $\left(n_{L}\right)$ rotational character. This is seen as a clockwise dextrorotation in optic axis direction towards the source of light by an observer.

Light traverses in the $b$-axis direction of a left-handed crystal in Figs. 3(c) and 3(d). In Fig. 3(c) the vectors $\mathbf{k}_{\mathbf{b}}, \mathbf{C}$ and $\mathbf{A}^{\prime}$ follow left-hand rule and give the axial vector $\mathbf{A}^{\prime}=\mathbf{k}_{\mathbf{b}} \mathbf{x} \mathbf{C}$ a counterclockwise $\left(n_{L}\right)$ rotation character. In Fig. 3(d) the vectors $\mathbf{k}_{\mathbf{b}}, \mathbf{A}$ and $\mathbf{C}^{\prime}$ follow the righthand rule and the dominant axial vector $\mathbf{C}^{\prime}$ in $\mathbf{C}^{\prime}=\mathbf{k}_{\mathbf{b}} \mathbf{x} \mathbf{A}$ will rotate the circularly polarized wave clockwise $\left(n_{R}\right)$ and it is seen as a counterclockwise levorotation in the optic axis direction towards the light source by an observer. This analysis results in the same optical chirality sense as experimentally measured in the absolute configuration of $\alpha-\mathrm{HgS}$ crystal in the space group $P 3_{2} 21$. [8] 


\section{Principal gyration tensor components and topological equivalences}

The components of the second electric moments in units e $\AA^{2}$, in TABLE III, can be converted to the principal gyration tensor components in units coulombcentimeter ${ }^{2}\left(\mathrm{Ccm}^{2}\right)$ through Eqs. (6) and (7):

$$
\begin{aligned}
& g_{11}=g_{22}=\Theta_{\mathrm{zz}}^{\prime} \cdot\left(-1.6021917 \cdot 10^{-19}\right) \cdot 10{ }^{16} \mathrm{Ccm}^{2} \\
& g_{33}=\left(\Theta^{\prime} \mathrm{xx}=\Theta_{\mathrm{yy}}^{\prime}\right) \cdot\left(-1.6021917 \cdot 10^{-19}\right) \cdot 10{ }^{16} \mathrm{Ccm}^{2}
\end{aligned}
$$

$g_{33}$ is the faster component with a higher magnitude than the lower components $g_{11}$ and $g_{22}$. It dominates and gives the correct sense for optical rotation in the both space groups of $\alpha-\mathrm{HgS}$ crystal. The values of them are written in TABLE IV.

Because the gyration tensor is an axial tensor of second rank, its components are axial vectors of second rank. [12] The two quadrics, presented in Figs. 2(a) and 2(b), have the following relationships between the ratios of the measure refractive indices, of the gyration tensor components, of the axial and polar vectors of second rank and of the refractive indices extracted from the axial vectors in the two space groups $P 3_{1} 21$ and $P 3_{2} 21$ of $\alpha-\mathrm{HgS}$ (cinnabar) crystal.
a) $P 3{ }_{1} 21: n_{e} / n_{o}=g_{33} / g_{22}=\mathbf{C}^{\prime} / \mathbf{B}^{\prime}=\left(\mathbf{k}_{\mathbf{a}} \times \mathbf{B}\right) /\left(\mathbf{k}_{\mathbf{a}} \times \mathbf{C}\right)=\mathbf{B} / \mathbf{C}=\Theta^{\prime}{ }_{\mathrm{yy}} / \Theta^{\prime}{ }_{\mathrm{zz}}=n_{L} / n_{R}$
b) $P 3_{2} 21: n_{e} / n_{o}=g_{33} / g_{11}=\mathbf{C}^{\prime} / \mathbf{A}^{\prime}=\left(\mathbf{k}_{\mathbf{b}} \mathbf{x} \mathbf{A}\right) /\left(\mathbf{k}_{\mathbf{b}} \mathrm{x} \mathbf{C}\right)=\mathbf{A} / \mathbf{C}=\Theta^{\prime} \mathrm{xx} / \Theta^{\prime}{ }_{\mathrm{zz}}=n_{R} / n_{L}$

The following parameters are equivalent in magnitudes:

In P3 $21: g_{33}=\Theta_{\mathrm{yy}}^{\prime}$ and $g_{22}=\Theta_{\mathrm{zz}}^{\prime}$ and in $P 3_{2} 21: g_{33}=\Theta^{\prime} \mathrm{xx}$ and $g_{11}=\Theta_{\mathrm{zz}}^{\prime}$, but due to symmetry $g_{33}=\Theta^{\prime} \mathrm{XX}=\Theta_{\mathrm{yy}}^{\prime}$ and $g_{11}=g_{22}=\Theta_{\mathrm{zz}}^{\prime}$ are consistent with both space groups and verify the invert relationship between the ratios of refractive indices and polar vectors of second rank in the two topologically equivalent quadrics of $\alpha-\mathrm{HgS}$ crystal.

\section{Optical rotation in $\alpha$-HgS crystal}

According to the theory of optical activity the plane polarized light splits up into the right- $\left(n_{R}\right)$ and left-handed $\left(n_{L}\right)$ circularly polarized waves, when it enters the crystal in optic axis direction, as supposed by Fresnel [1], [12]. The waves are in phase at the entrance. They traverse with slightly different wavelengths depending on the left- and right-handed character 
of the crystal. The faster component is ahead in phase at the emergency and the sense of rotation will be the same as the sense of the faster of the two circularly polarized components.

In the space group $P 3{ }_{1} 21$ the rotational senses are counterclockwise in the dominant $\mathbf{C}^{\prime}\left(n_{L}\right)$ and clockwise in the slower $\mathbf{B}^{\prime}\left(n_{R}\right)$ component, respectively, and in the space group $P 3_{2} 21$ the difference between the dominant $\mathbf{C}^{\prime}\left(n_{R}\right)$ and the slower $\mathbf{A}^{\prime}\left(n_{L}\right)$ components gives the magnitude and the sense of optical rotation per unit path in the crystals according to Eq. (8).

$$
\phi=\frac{\pi \cdot\left(n_{L}-n_{R}\right)}{\lambda} \text { radians. }
$$

The principal refractive indices are usually presented for a plate of crystal with a thickness of 1 $\mathrm{cm}$ and the optical rotation with the thickness of $1 \mathrm{~mm}$, cut normal to an optic axis, where a plane polarized light passes along the optic axis, which is free of birefringence. The magnitude and the sense optical rotation are expressed in the two space groups $P 3_{1} 21$ and $P 3_{2} 21$ of $\alpha-\mathrm{HgS}$ crystal, with $\lambda=6328 \cdot 10^{-8} \mathrm{~cm}$, according to the Eqs. (9) and (10), respectively, in the righthanded system of coordinate axes and summarized in TABLE IV.

$$
\begin{aligned}
& \phi=\frac{0.1 \cdot \pi \cdot\left(g_{33}-g_{22}\right)}{\lambda} \mathrm{rad} . \mathrm{mm}^{-1} \\
& \Rightarrow \phi=+5.5656 \mathrm{rad} . \mathrm{mm}^{-1}=+318.9^{\circ} \mathrm{mm}^{-1} . \\
& \phi=\frac{0.1 \cdot \pi \cdot\left(g_{11}-g_{33}\right)}{\lambda} \mathrm{rad} . \mathrm{mm}^{-1} \\
& \Rightarrow \phi=-5.5656 \mathrm{rad} . \mathrm{mm}^{-1}=-318.9^{\circ} \mathrm{mm}^{-1} .
\end{aligned}
$$

Table IV. Gyration tensor components and magnitude and sense of optical rotation in the unit cells of two space groups of $\alpha-\mathrm{HgS}$ crystal. The experimentally measured value: $\phi=-320^{\circ} \mathrm{mm}^{-1}$ [9] and the calculated value from the atomic polarizabilities: $\phi=-310^{\circ} \mathrm{mm}^{-1}[10]$ for a levorotatory crystal with $\lambda=6328 \AA$.

\begin{tabular}{|c|c|c|c|c|}
\hline Space group & $\begin{array}{c}\left(g_{11}=g_{22}\right) \cdot 10^{-3} \\
\left(\mathrm{Ccm}^{2}\right)\end{array}$ & $\begin{array}{c}g_{33} \cdot 10^{-3} \\
\left(\mathrm{Ccm}^{2}\right)\end{array}$ & $\begin{array}{c}\phi \\
\left(\mathrm{rad} . \mathrm{mm}^{-1}\right)\end{array}$ & $\begin{array}{c}\phi \\
\left(\mathrm{mm}^{-1}\right)\end{array}$ \\
\hline$P 3_{12} 1$ & 9.31630 & 10.43735 & +5.5656 & +318.9 \\
\hline$P 3_{2} 21$ & 9.31630 & 10.43735 & -5.5656 & -318.9 \\
\hline
\end{tabular}

\section{Discussion}

The purpose of this study was to find out a simple and reliable explanation for the optical rotation in the enantiomorphic pairs of $\alpha-\mathrm{HgS}$ crystal. The crystal structure of $\alpha-\mathrm{HgS}$ has very definite helices of covalently bounded atoms. In this study the obtained sense of optical 
rotation is opposite to the rotational sense of the atomic helix and it is in agreement with the experimental measurement. The magnitude and sense of optical rotation arise from the unit cell information in the crystal. Both $\mathrm{Hg}$ and $\mathrm{S}$ atoms with their charges in a chain create a system of electric moments. The axial vectors with their handedness and magnitudes drive and guide the rotation sense of the electric displacement vectors of the two circularly polarized components of light, when they propagate through the crystal. The dominant components are ahead and determine the sense of optical chirality in the enantiomorphs of the $\alpha-\mathrm{HgS}$ crystal.

$\alpha$-HgS crystal in the space group $P 3_{2} 21$ was tested levorotatory and in accordance with this work it is a left-handed crystal. The mirror image of it is a right-handed dextrorotatory enantiomorph in the space group $P 3{ }_{12} 1$ with an anticlockwise rotation sense of atomic helix, which must have the absolute crystal structure of $\alpha-\mathrm{HgS}$, because light always propagates in the $a$-axis direction of the right-handed crystal in the measurement of refractive indices. The sense of optical rotation has no correlation to the rotational sense of the helical chain of the atoms in the $\alpha-\mathrm{HgS}$ crystal. It is possible to find out other atomic helices in the crystal structures with the same sense as that of optical rotation in favor of the other type of theoretical descriptions, but that can sometimes distort the truth.

The calculations presented in this paper are transparent and can be approved with a programable pocket calculator by any reader, who is interested in this type of computations. There are existing several simple chemical compounds, where the atomic charges can be iterated or refined with the anomalous X-ray diffraction technique. The knowledge of the absolute crystal structure and atomic net charges creates the primary conditions to discover new explanations for unclear chemical or physical properties of crystals.

\section{Acknowledgement}

The University of Uppsala is acknowledged with pleasure for the offered possibility to make some research in chemical and physical properties of crystals.

\section{References}

[1] Fresnel, A., (1824). Bull. Soc. Philomath. Paris, pp. 147-158.

[2] Born, M., (1922). Z. Phys. 8, 390 - 417. 
[3] Hylleraas, E.A., (1927). Z.Phys. 44, 871.

[4] Condon, E.U., (1937). Rev.Mod. Phys., 9, 432-457.

[5] Cotton, F.A. and Wilkinson, G., (1972). Advanced Inorganic Chemistry, $3^{\text {rd }}$ ed. Interscience, New York, 1972.

[6] Lamure, J., Brusset, H., (1962). Mercure. In: Nouveau Traité de Chimie Minérale, Tome V, Pascal P. Ed., 733-797.

[7] Auvray, P. and Genet. F., (1973). Bulletin de la Societe Francaise de Mineralogie et de Cristallographie, 96, 218-219.

[8] Auvray, P., (1976). Bulletin de la Societe Francaise de Mineralogie et de Cristallographie, 99, 373-379.

[9] Ayrault, I.B., Lefin, F., Langlaois, H., Toudic. Y., Palmier, J.F., (1972). Optics Communications, Volume 5, Issue 4, 239-243.

[10] Devardajan, V., and Glazer, A., M., (1986). Acta Cryst. A42, 560-569.

[11] Glazer, A.M. \& Stadnicka, K. (1986). J. Appl. Cryst. 19, 108-122.

[12] Nye, J.F. (1972)., Physical Properties of Crystals. Oxford: Clarendon Press.

[13] Buckingham, A.D., (1959). Q.Rev. Chem.Soc. 13,183.

[14] Buckingham, A.D., (1970). Physical Chemistry. An Advanced Treatise. Vol. IV, p.349. New York: Academic Press.

[15] Farrugia, L. J., (1997). ORTEP-3 for Windows. J. Appl. Cryst. 30, 565.

[16] W. L. Bond, G. D. Boyd and H. L. Carter Jr., (1967). J. Appl. Phys., 38, 4090-4091 (https://RefractiveIndex.INFO).

[17] Karppinen. M., (2015). Acta Crystallogr. Sec.B 71, 334-341.

[18] Karppinen, M., (2020). J.Appl.Crystallogr. 53, 1252-1256.

[19] Karppinen, M., (2022). SCIREA Journal of Chemistry. (https://doi.org/10.54647chemistry 15232) 